\title{
Improving maternal mortality reporting at the community level with a 4-question modified reproductive age mortality survey (RAMOS)
}

\author{
Julia Geynisman ${ }^{\mathrm{a}}$, Andrew Latimer ${ }^{\mathrm{a}}$, Anthony Ofosu ${ }^{\mathrm{b}}$, Frank W.J. Anderson ${ }^{\mathrm{c}, *}$ \\ a University of Michigan Medical School, Ann Arbor, USA \\ b Ghana Health Service and Ministry of Health, Accra, Ghana \\ c Department of Obstetrics and Gynecology, University of Michigan, Ann Arbor, USA
}

\section{A R T I C L E I N F O}

\section{Article history:}

Received 26 October 2010

Received in revised form 19 January 2011

Accepted 16 March 2011

\section{Keywords:}

Ghana

International health

Maternal mortality

Reproductive age mortality survey (RAMOS)

\begin{abstract}
A B S T R A C T
Objective: To investigate the identification of maternal deaths at the community level using the reproductive age mortality survey (RAMOS) in all households in which a women of reproductive age (WRA) died and to determine the most concise subset of questions for identifying a pregnancy-related death for further investigation. Methods: A full RAMOS survey was conducted with the families of 46 deceased WRA who died between 2005 and July 2009 and was compared with the cause of death confirmed by the maternal mortality review committee to establish the number of maternal mortalities. The positive predictive value (PPV) of each RAMOS question for identifying a maternal death was determined. Results: Compared with years of voluntary reporting, active surveillance for maternal deaths doubled their identification. In addition, 4 questions from the full RAMOS have the highest PPV for a maternal death including the question: "Was she pregnant within the last 6 weeks?" which had a 100\% PPV and a 100\% negative predictive value. Conclusion: Active identification of maternal mortality at the community level by using a 4-question modified RAMOS that is systematically administered in the local language by health workers can increase understanding of the extent of maternal mortality in rural Ghana.

c 2011 International Federation of Gynecology and Obstetrics. Published by Elsevier Ireland Ltd. All rights reserved.
\end{abstract}

\section{Introduction}

Investigative studies routinely uncover significant underreporting of maternal mortality in low-resource countries; in rural areas, and at the community level, maternal mortality is notoriously difficult to identify [1-3]. National birth and death certification processes are variably implemented, and data collection frequently does not extend into the rural communities where many maternal deaths occur. Methods based on national surveys are used infrequently, and hospital-based studies include only deaths to women with access to health facilities. Other deaths are missed when social norms repress open dialogue about reproductive health, or when a deceased woman was already marginalized by her community. Despite the barriers, it is crucial to find consistently effective ways to identify, report, and investigate maternal deaths at the community level. Acknowledging and studying the factors related to the death can serve as witness to these women's deaths, and possibly can be mined for answers to the underlying causes of these deaths and be incorporated into solutions and policies [4].

\footnotetext{
* Corresponding author at: Medical School, Health Behavior \& Health Education, 3822 SPH I, Office 2029, 1415 Washington Heights, Ann Arbor, MI 48109-2029, USA. Tel.: +1 734615 4396; fax: +1 7346479727 .

E-mail address: fwja@umich.edu (F.W.J. Anderson).
}

Examining deaths to all women of reproductive age (WRA) is one strategic method for identifying maternal deaths because they will always be a subset of this group. The reproductive age mortality survey (RAMOS) is a 39-question survey, administered to the surviving spouse or next of kin, that assesses signs and symptoms related to the death, which include general and reproductive health questions to determine whether the decedent was pregnant at the time of death, the outcome of the pregnancy, the use of family planning, and symptoms such as vaginal bleeding and abdominal or pelvic pain.

The RAMOS has been used in assessments of maternal mortality in hospitals in several low-resource countries, including Surinam, Tanzania, Gambia, Mozambique, and Taiwan [1,2,5-8]. In all of these studies, the use of the RAMOS increased the identification of maternal deaths compared with hospital records and official government estimates. In Mozambique, use of the RAMOS increased the identification of maternal deaths in hospital, but it did not include the outcomes of the $60 \%$ of deliveries that occurred outside the healthcare facilities [6]. Likewise, in Surinam, use of the RAMOS into deaths in 5 hospitals over 9 years found that the number of maternal deaths identified was 30 percent higher than the officially reported number of maternal deaths for the whole country [8].

The RAMOS was used in Ghana in 2002 by the Ghana Health Service (GHS) in an extensive maternal mortality review in selected areas of Accra; the survey identified almost twice the number of 
maternal deaths as the officially reported number of maternal deaths [9]. In 2007, the GHS coordinated a comprehensive study that compared the ability of sisterhood studies versus the use of RAMOS followed by verbal autopsies to identify maternal deaths in hospital using several metropolitan areas as their study population. The study found that the estimate of the pregnancy-related mortality ratio from the sibling history was 378 per 100000 live births, whereas the estimate of the maternal mortality ratio for the same time period from RAMOS plus verbal autopsies was 580 per 100000 live births [10].

The relative success of the use of the RAMOS in hospitals prompted an interest in using this survey to identify maternal deaths in rural Ghana. The Sene district is a rural region in northeast Ghana that covers 8586 square kilometers and has a population of about 100000 . Notification of maternal mortality in the Sene district is by voluntary reporting of maternal deaths by family members, or by public health personnel who make visits to the village and might be notified by a community elder about a death of a pregnant woman. A public health nurse is then dispatched to conduct an investigation, by using a verbal autopsy and medical records to review factors related to the death. Maternal mortality review committees routinely meet in the Sene district to review data from the investigation to establish a final diagnosis and to determine systems and community issues that may have contributed to the death.

The voluntary reporting system in the Sene district was considered to result in underreporting of maternal deaths. In 2008, a pilot program that investigated deaths to WRA using the RAMOS was implemented. Community health workers were actively encouraged and given an incentive to report any deaths of a WRA (10-49 years old) after which hospital staff members were dispatched to complete a full RAMOS with the next of kin. The RAMOS was reviewed by the district medical officer and any answers that suggested a pregnancyrelated death (for example vaginal bleeding, seizures, or abdominal pain) triggered the hospital staff to conduct a verbal autopsy and to determine whether this was a maternal death.

The use of the full RAMOS at the community level is limited by the baseline educational level of the rural population and the implicit requirement of the survey for some literacy and understanding of the physiological and temporal details of a death. The aim of the present study was to review the experience of the Sene district with the RAMOS to determine whether it identified more maternal deaths and whether it could be shortened to a simpler subset of questions that can identify a community level pregnancy-related death for further investigation.

\section{Materials and methods}

The present study consisted of a review of cases of known maternal deaths and deaths to WRA from non-obstetric causes reported in the Sene district between January 2005 and July 2009. The record reviews were conducted between July 1 and August 15, 2009. The records from all maternal mortality reviews from 2005 to 2009 were examined, in addition to data from the RAMOS conducted in 2008. For maternal deaths in years other than 2008 and for all other confirmed maternal deaths that did not have an accompanying RAMOS survey, the project staff arranged to meet with the families of the deceased woman, traditional birthing attendant, or the local health worker; verbal consent was obtained and the RAMOS was administered. The questionnaire was administered in a home or clinic and was translated into the local dialect. The study was approved by the University of Michigan Institutional Review Board and the Ghana Health Service Ethical Review Board; no incentives were given to participants.

RAMOS results and those of maternal mortality reviews were available for 46 WRA who died during the period of review. Thirty deaths were confirmed as maternal deaths and 16 were non-maternal deaths as determined by RAMOS. Non-maternal deaths served as negative controls.

The RAMOS questionnaire was reviewed, and the 28 nonreproductive health questions were excluded from the analysis. The remaining 11 questions represented information that could potentially correlate with a maternal death (Table 1 ).

The results of the maternal mortality review were used as the gold standard for the diagnosis of a maternal death and were used to calculate the positive predictive values (PPVs) and negative predictive values (NPVs) of each selected RAMOS question. The PPVs were calculated by dividing the positive responses for that question among maternal death cases by all positive answers (true positives/[true positives + false positives]). The NPVs were calculated by dividing the number of negative responses to each question from non-maternal death cases by all negative answers (true negatives/[true negatives + false negatives]). Indefinite answers were not counted as positive or negative answers.

\section{Results}

In 2005, 2006 and 2007, 7, 3, and 7 maternal deaths were identified, respectively. In 2008, the year of active surveillance of deaths of WRA, 13 maternal deaths were identified. In 2009, when the district reverted back to voluntary reporting, only 7 maternal deaths were reported ( 2 of which occurred in the study period). Active surveillance with RAMOS in 2008 also identified 16 non-maternal deaths of WRA, which serve as negative controls. Of the 32 maternal mortalities that occurred during this time, 2 families could not be located; therefore, the remainder of the data addresses 30 maternal mortalities that were investigated during the period studied. Of the 30 women who died, 22 women died of direct maternal deaths and 8 women died of indirect maternal deaths. The most common causes of direct maternal death, as determined by the maternal mortality review process, were postpartum hemorrhage (50\%) followed

Table 1

Positive predictive value of 11 questions from the RAMOS.

\begin{tabular}{|c|c|c|c|c|}
\hline Question from the full RAMOS & $\begin{array}{l}\text { Positive responses out of all maternal } \\
\text { deaths }(n=30) \text { (true positives) }\end{array}$ & $\begin{array}{l}\text { Negative responses out of all confirmed } \\
\text { non-maternal deaths }(n=16) \text { (true negatives) }\end{array}$ & $\begin{array}{l}\text { PPV } \\
(\%)\end{array}$ & $\begin{array}{l}\text { NPV } \\
(\%)\end{array}$ \\
\hline Did she have pain in her stomach or abdomen? & 6 & 14 & 75.0 & 37.8 \\
\hline Was she bleeding from the vagina? & 15 & 14 & 88.2 & 51.9 \\
\hline Is her last child less than 1 year old? & 3 & 4 & 42.9 & 12.5 \\
\hline Has she been pregnant since her last child was born? & 20 & 1 & 95.2 & 56.2 \\
\hline Has she ever had an induced abortion? & 4 & 1 & 80.0 & 38.4 \\
\hline Did she stop using family planning? & 28 & 13 & 69.0 & 100 \\
\hline $\begin{array}{l}\text { During her illness, did she ever collapse, and if so, } \\
\text { was it within } 7 \text { days of her death? }\end{array}$ & 3 & 0 & 100 & 37.0 \\
\hline $\begin{array}{l}\text { Did she ever lose consciousness, and if so, was } \\
\text { it within } 7 \text { days of her death? }\end{array}$ & 2 & 1 & 66.0 & 34.8 \\
\hline Was she pregnant when she died? & 21 & 0 & 100 & 68.0 \\
\hline Was she recently pregnant (within the last 6 weeks)? & 30 & 0 & 100 & 100 \\
\hline Did she live less than a year after her last pregnancy ended? & 30 & 3 & 91.0 & 100 \\
\hline
\end{tabular}

Abbreviations: NPV, true negatives divided by all negative answers; PPV, true positives divided by all positive answers; RAMOS, reproductive age mortality survey. 
Table 2

Causes of maternal deaths (2005-2009).

\begin{tabular}{lc}
\hline Causes of death & No. of deaths \\
\hline Direct maternal deaths & 11 \\
Postpartum hemorrhage & 4 \\
Unsafe abortion & 3 \\
Prolonged obstructed labor & 2 \\
Anemia + pre-eclampsia & 1 \\
Prepartum + postpartum hemorrhage & 1 \\
Eclampsia & 22 \\
Total & \\
Indirect maternal deaths & 2 \\
Anemia & 1 \\
Cardiac arrest secondary to labor & 1 \\
Malaria + anemia & 1 \\
Sickle cell crisis & 1 \\
Liver failure & 1 \\
Hemorrhage & 1 \\
Cholera & 8 \\
Total & 16 \\
Non-maternal deaths & 46 \\
All deaths to women of reproductive age
\end{tabular}

by unsafe abortion (18\%). Pre-eclampsia and eclampsia accounted for $10 \%$ of the causes of all maternal deaths. The causes of indirect maternal death vary widely but anemia was the most common cause in this cohort (25\%). The remaining causes of death are shown in Table 2.

The average age of the women at the time of death was 28 years (range 13-47 years). In the maternal mortality group, there were 21 perinatal births and 9 live births. Two-thirds of the women died in the community, whereas one-third of the women died in health centers. At the time of their death, the average gravidity of the women was 3.5, and the average parity was 2.8 .

The respondents to the RAMOS survey were most often the husband $(12 / 30)$ or the mother (9/30). In the remaining cases, the survey was answered by a first degree relative, health worker, or traditional birth attendant.

The PPVs and NPVs for each of the 11 questions are listed in Table 1. The questions that yielded both high PPVs and high NPVs were "was she recently pregnant (within the last 6 weeks)?" and "did she live less than a year since her last pregnancy ended?" Several other questions elicited a high PPV: "was she pregnant when she died?" (100\% PPV); "did she ever have an induced abortion?" (80\% PPV); "was she bleeding from the vagina?" ( $88 \%$ PPV). We analyzed the 2008 data separately and did not find a significant difference between the results of that year alone and the results of all years combined.

\section{Discussion}

The results from the present pilot program show that active surveillance of deaths of WRA at the community level in the Sene district of Ghana more than doubled the average number of reported maternal deaths per year compared with years in which voluntary reporting was the norm. These results also suggest that a subset of questions with high PPVs can accurately identify pregnancy-related deaths from the pool of WRA who have died that require further investigation. This "modified" RAMOS could be used as a communitybased screening tool on all deaths to WRA; a positive response to any of the 4 questions suggested by our data to be predictive would trigger a verbal autopsy protocol. This procedure would require fewer resources and could easily be included in the duties of community health workers.

The present study was only able to calculate the PPV and NPV for each question. Because the confirmatory diagnostic process of verbal autopsies and mortality reviews were not performed on cases in which a maternal death was not suspected, the sensitivity and specificity of these questions could not be determined. Conducting a verbal autopsy on each case where 1 of the relevant reproductive questions was answered affirmatively would allow us to determine the "true and false negatives" needed to calculate sensitivity and specificity. Ectopic pregnancy deaths, especially, would be a significant source of false negatives, even if they could be determined by verbal autopsy. Non-obstetric vaginal bleeding and other causes of sudden death could contribute to false positives.

Not surprisingly, the present analysis found that the question about abortion has a high PPV because of the high rate of mortality from this procedure. Although elective abortions are allowed in Ghana for medical and psychological health reasons, the lack of facilities and trained personnel, and the social and religious stigmatization that accompanies this procedure, pushes women to seek unsafe abortions from family members or other community members [11]. Other reproductive questions were less useful because of their cultural or contextual content. For example, a question such as "did she stop using family planning?" was ineffective for screening for maternal deaths because very few of the households visited reported use of family planning.

The study is limited by the retrospective nature of the data collection for RAMOS done outside 2008, which in some cases may have been 5 years after the death of the woman. In these cases underreporting of a death associated with a pregnancy may have been expected. This does not appear to be the case. A separate analysis of the 2008 data demonstrated the same trends in predicative values as the entire dataset.

The use of incentives in 2008 to encourage use of the lengthy RAMOS form is inextricable from the result that there was increased identification of all deaths of WRA in 2008. We are unable to show statistically that without incentives the same number of RAMOS would have been collected; however, we expect that if health workers can perform the 2-pronged screening and confirmatory process, identification of pregnancy-related deaths will remain high without financial incentives.

This study of the use of the RAMOS questionnaire in a community setting to identify potential maternal deaths in the rural population of Sene District, Ghana, showed that asking whether a woman had been pregnant within the last 6 weeks identified $100 \%$ of the maternal deaths with a 100\% PPV and 100\% NPV. This should be the first question in the shortened RAMOS. Because most maternal deaths in the district are the result of hemorrhage or abortion, we recommend that the second question should be, "Was she bleeding from the vagina?"; the third question should be "Was she pregnant when she died?", and the fourth question should be "Has she ever had an induced abortion?" This 4-item questionnaire should be distributed to community health workers who are trained to use this questionnaire when assessing the death of a WRA in their catchment area. If any of these questions is answered positively, further investigation into the cause of death should be initiated. Active surveillance should be coupled with ongoing research into its impact on the reporting and awareness of maternal mortality in Sene district.

\section{Conflict of interest}

The authors have no conflicts of interest.

\section{References}

[1] Olsen BE, Hinderaker SG, Lie RT, Bergsjø P, Gasheka P, Kvåle G. Maternal mortality in northern rural Tanzania: assessing the completeness of various information sources. Acta Obstet Gynecol Scand 2002;81(4):301-7.

[2] Kao S, Chen LM, Shi L, Weinrich MC. Underreporting and misclassification of maternal mortality in Taiwan. Acta Obstet Gynecol Scand 1997;76(7):629-36.

[3] Campbell OMR, Graham WJ. Measuring Maternal Mortality and Morbidity: Levels and Trends. Maternal and Child Epidemiology Unit Publication No. 2. London: London School of Hygiene and Tropical Medicine; 1990.

[4] WHO. Beyond the numbers: reviewing maternal deaths and complications to make pregnancy safer. www.who.inthttp://whqlibdoc.who.int/publications/2004/ 9241591838.pdf. Published 2004. 
[5] Walraven G, Telfer M, Rowley J, Ronsmans C. Maternal mortality in rural Gambia: levels, causes and contributing factors. Bull World Health Organ 2000;78(5): 603-13.

[6] Songane FF, Bergström S. Quality of registration of maternal deaths in Mozambique: a community-based study in rural and urban areas. Soc Sci Med 2002;54(1):23-31.

[7] Fortney J, Gadalla S, Saleh S, Susanti I, Potts M, Rogers SM. Causes of death to women of reproductive age in two developing countries. Popul Res Policy Rev 1987;6:137-48.

[8] Mungra A, van Bokhoven SC, Florie J, van Kanten RW, van Roosmalen J, Kanhai HH. Reproductive age mortality survey to study under-reporting of maternal mortality in Surinam. Eur J Obstet Gynecol Reprod Biol 1998;77(1):37-9.
[9] Zakariah AY, Alexander S, van Roosmalen J, Buekens P, Kwawukume EY, Frimpong P. Reproductive age mortality survey (RAMOS) in Accra, Ghana. Reprod Health 2009;6:7.

[10] Ghana Statistical Service (GSS), Ghana Health Service (GHS), Macro International. Ghana Maternal Health Survey 2007. www.usaid.govhttp://pdf.usaid.gov/ pdf_docs/PNADO492.pdf. Published 2009.

[11] Morhe ESK, Morhe RAS, Danso KA. Attitudes of doctors toward establishing safe abortion units in Ghana. Int J Gynecol Obstet 2007;98(1):70-4. 